\title{
A rare complication of mediastinal mass biopsy
}

\author{
Kruti Patel ${ }^{1 *}$, Ankur Shah ${ }^{1}$, Ryan Kahl ${ }^{4}$, Asim Ahmed ${ }^{3}$, Sarah Keller ${ }^{1}$, Robert Palma ${ }^{1}$ and Lucia Chowdhury ${ }^{2}$ \\ ${ }^{1}$ Department of Internal Medicine, Doctors Hospital, Columbus, Ohio, USA \\ ${ }^{2}$ Department of Pulmonary and Critical Care Medicine, Doctors Hospital, Columbus, Ohio, USA \\ ${ }^{3}$ Department of Cardiology and vascular disease, Doctors Hospital, Columbus, Ohio, USA \\ ${ }^{4}$ Department of Internal Medicine, Saint John's Macomb-Oakland Hospital, Warren, Michigan, USA
}

27 year old female with history of atherosclerotic cardiovascular disease and remote myocardial infarction, asthma and provoked deep vein thrombosis (completed anticoagulation) presented with complaints of worsening chest pain and dyspnea for two days. She denied fever, chills, productive cough or sick contacts. She admitted to 70-pound unintentional weight loss over 7 months, decreased appetite, night sweats, diarrhea, and palpitations.

Vital signs showed heart rate 95, blood pressure 113/74 $\mathrm{mmHg}$, respiratory rate $14, \mathrm{SpO} 295 \%$ on room air. A comprehensive metabolic panel and complete blood count were normal. EKG showed normal sinus rhythm with left ventricular hypertrophy and no ST changes. Urine drug screen was positive for cannabinoid only. Cardiac markers were negative. TSH was $0.01 \mathrm{mclU} / \mathrm{mL}(0.31-5.00 \mathrm{mclU} / \mathrm{mL})$.

Hospital Day 1: Chest x-ray showed a small density projecting over the right upper lung at the intersection of anterior $3^{\text {rd }}$ and posterior $6^{\text {th }}$ ribs. Follow up with CT pulmonary angiogram showed indeterminate homogenous solid mass within the anterior superior mediastinum along the aortic arch measuring approximately $5.5 \mathrm{~cm} \mathrm{x}$ $5.3 \mathrm{~cm} \times 2.4 \mathrm{~cm}$ in craniocaudal, transverse and AP dimensions. Mass was not continuous with the thyroid and did not demonstrate cystic or calcified components [Figures 1 and 2]. Thyromegaly without focal lesion was noted on CT imaging. Heart was normal in size without pericardial effusion and a collapsible Inferior vena cava [Figure 3]. US thyroid revealed bilateral Thyromegaly with hypervascularity and no discrete nodules.

Hospital Day 2: Due to suspicion for possible lymphoma or thymoma, Interventional radiology and Oncology were consulted to obtain biopsy of anterior mediastinal mass for further evaluation [Figure 4].

Shortly after returning from CT guided-needle biopsy of mediastinal mass, the patient developed nausea, profuse vomiting and became unresponsive. Upon arrival of Rapid Response Team, vital signs were heart rate 150 , blood pressure $65 / 50 \mathrm{mmHg}$, RR 20, SpO2 95\% on 6 L Oxygen. EKG showed sinus tachycardia without ST changes. Patient was given $1 \mathrm{~L}$ normal saline bolus and packed red blood cells were emergently prepared given concern for pulmonary artery injury as complication of biopsy. Bedside Ultrasound revealed pericardial effusion, non-collapsible inferior vena cava, and ventricular interdependence demonstrating tamponade physiology [Figures 5-7]. Patient was intubated for airway protection and cardiothoracic surgery was emergently consulted for pericardiocentesis and evacuation of suspected mediastinal hematoma.Chest $\mathrm{x}$-ray confirmed significant mediastinal widening suspicious for mediastinal hematoma [Figure 8].

Review of laboratory studies once patient was transferred to Intensive Care Unit showed TSH $0.01 \mathrm{mclU} / \mathrm{mL}(0.31-5.00 \mathrm{mclU} / \mathrm{mL})$ with reflex Free T4 $>4.2$ ng/dL (0.7-1.7 ng/dL) and T3 > 651 (71-170ng/ dL).Acetylcholine Receptor Binding Antibody was negative. BurchWartofsky score $>45$, classifying patient as having Thyroid Storm.

Emergent exploratory median sternotomy was performed with intraoperative administration of propylthiouracil, hydrocortisone sodium succinate and lugol iodine solution. Mediastinal exploration revealed a large anterior mediastinal mass with hemorrhage which was removed. Evidence of penetration of pericardial wall was noted; $150 \mathrm{cc}$ blood and clot were evacuated. Straight chest tubes were placed into each pleural cavity. Patient was transferred back to ICU in stable condition.

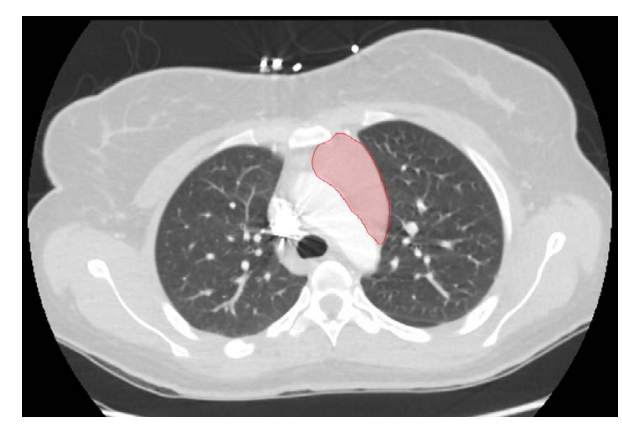

Figure 1. Intermediate homogenous solid mass within superior mediastinum measuring 5.5 $\mathrm{cm} \times 5.3 \mathrm{~cm} \times 2.4 \mathrm{~cm}$

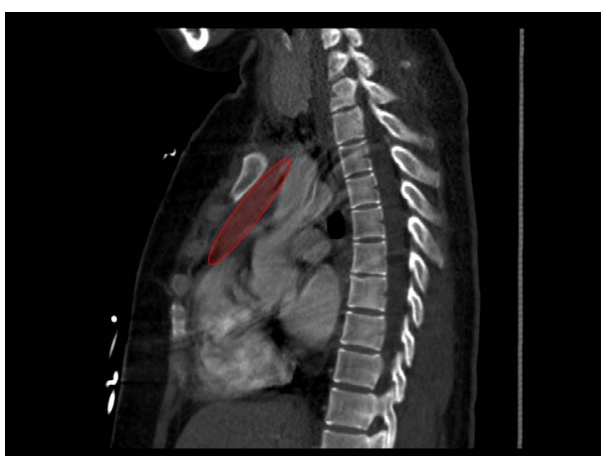

Figure 2. Representation of mass in sagittal view.

Correspondence to: Kruti Patel,Department of Internal Medicine, Doctors Hospital, Columbus, Ohio, USA, E-mail: Kruti.Patel@ohiohealth.com

Received: March 15, 2017; Accepted: March 24, 2017; Published: March 27, 2017 


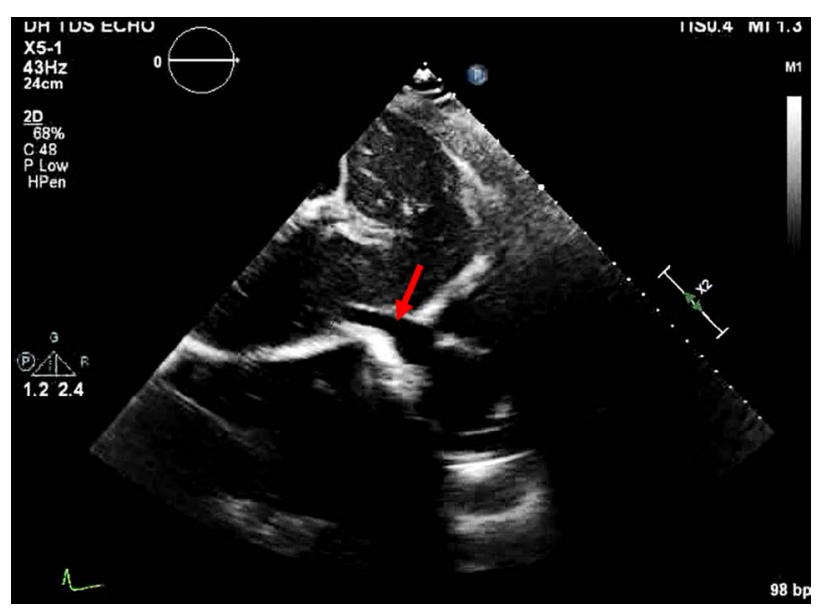

Figure 3. Collapsible IVC without signs of pericardial effusion.

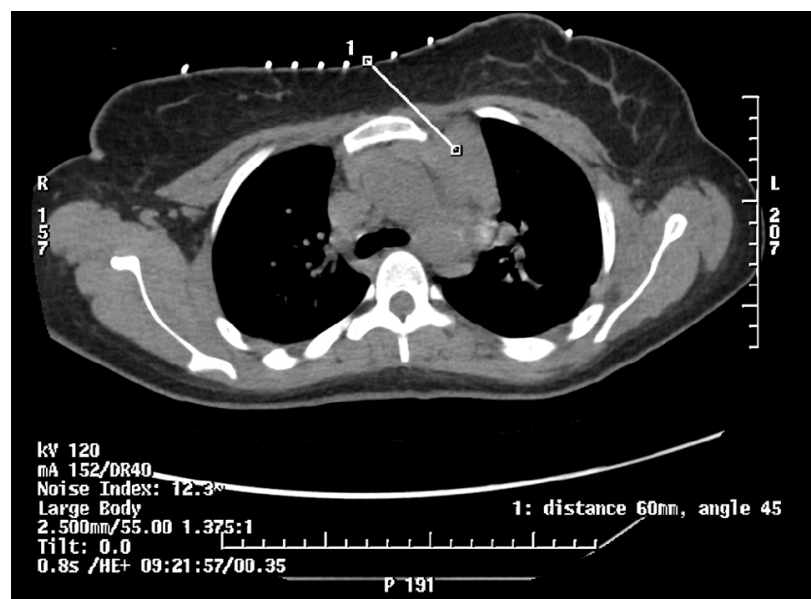

Figure 4. CT guided 18-gauge core biopsy to obtain abnormal soft tissue density in the anterior left upper mediastinum.

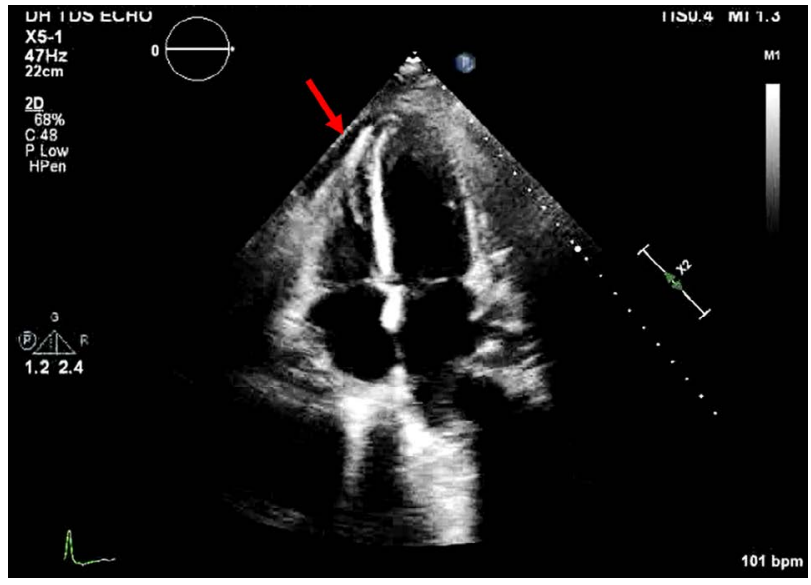

Figure 5. Axial 4 chamber view with pericardial effusion.

Pathologic examination of anterior mediastinal mass identified a bilobed thymus weighing 90.8 grams. Histologic analysis of tissue biopsy of mediastinal mass showed benign thymic hyperplasia likely secondary to untreated hyperthyroidism.

Patient was discharged home in stable condition with propranolol, propylthiouracil and instructions to follow up with endocrinology.

\section{Discussion}

Image guided mediastinal biopsy is a generally safe and minimally invasive procedure. There are multiple approaches and variety of imaging techniques - Ultrasound and Computed Tomography (CT) guided being the most common methods. Imaging technique is selected based on certain characteristics: clinical circumstances, location of the mass, size of the mass and underlying conditions of the specimen being biopsied. Anatomical and technical aspects, vascular components/vital organs, needle selection and pathologic considerations are considered prior to selection of approach.

Direct mediastinal approaches include para-vertebral, suprasternal, trans-pulmonary and extra pleural [1]. CT guided percutaneous biopsy has several advantages over other alternative techniques. It allows for precise localization of target lesion and access to mediastinal compartments which are not readily accessible by mediastinoscopy or endobronchialbiopsy [2]. US guided percutaneous biopsy has some benefits when compared to CT guided, most notably the ability to be performed in real time with continuous monitoring of needle during advancement and sampling. Multiple oblique paths are also easily accessed with US guidance and its portability portends the ability to perform biopsies at bedside in critically ill patients $[1,3]$.

CT guided biopsy provides detailed imaging and multiplanar reformations to help assist in avoiding puncture of vascular structures and vital organs in the thoracic cavity. CT guided percutaneous transthoracic needle biopsy is less invasive than mediastinoscopy and requires only local anesthesia. The few contraindications for use of percutaneous transthoracic needle biopsy are suspicion of hydatid cyst (absolute contraindication), coagulopathy, and severely compromised pulmonary function including bullous emphysema, pulmonary hypertension, or vascular tumor [3].

In our patient, an anterior mediastinal mass located in left parasternal and retrosternal region was identified. For an anterior or middle mediastinal mass in a patient without contraindications, the parasternal approach with CT guidance is most commonly used. In the parasternal approach, the needle is inserted through the parasternal muscles and mediastinal fat into the target lesion. Multiple scans are obtained between incremental needle advancement to check trajectory to ensure internal mammary vessels are not in path. A 22 gauge needle can be used inject saline solution or dilute contrast medium to widen

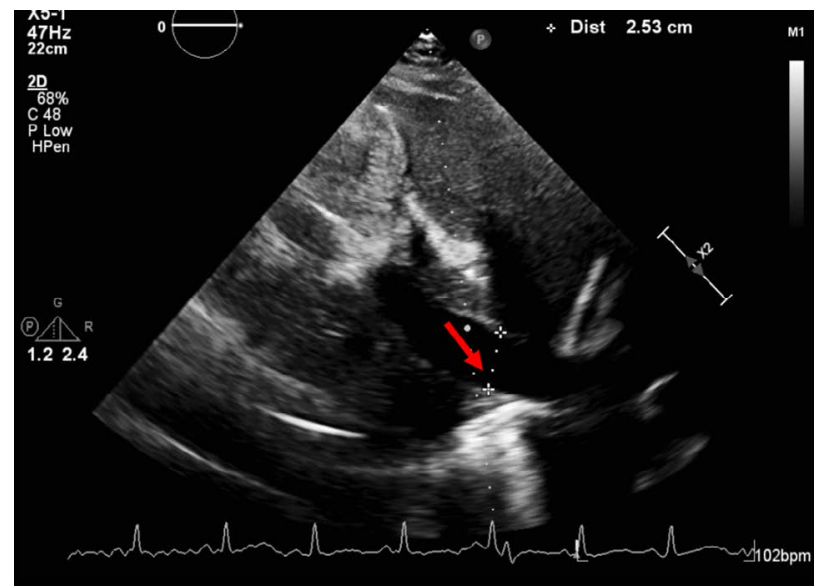

Figure 6. Non-collapsible Inferior Vena Cava. Presence of a dilated IVC with less than $50 \%$ collapse on forced inspiration correlates with elevated central venous pressure and confirms tamponade physiology. 


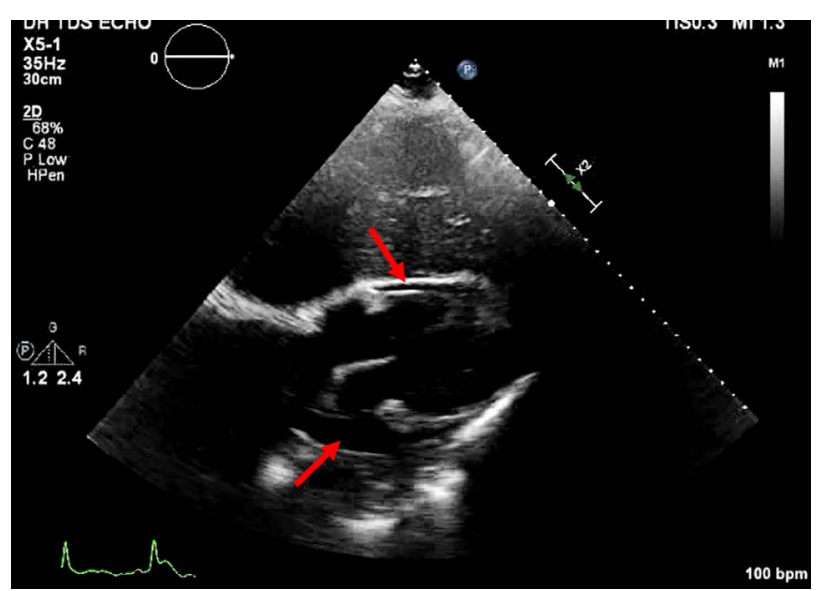

Figure 7. Subcostal view with pericardial effusion.

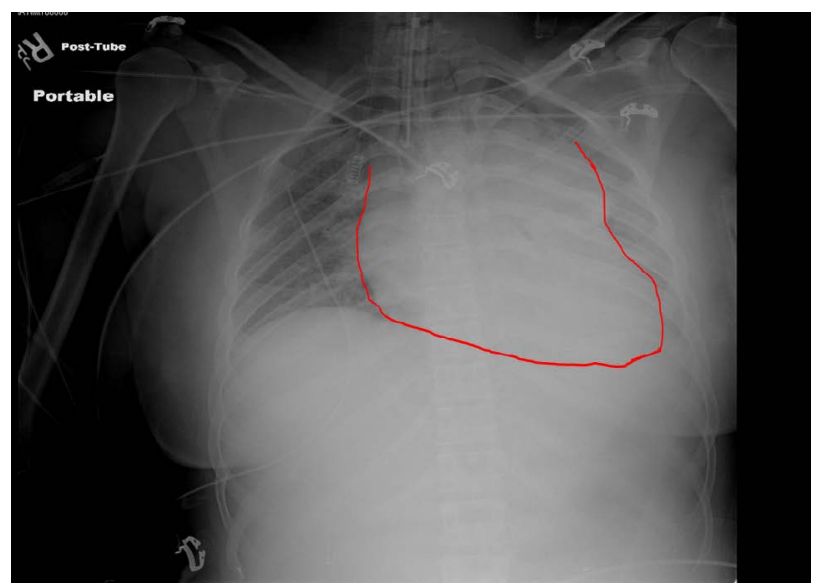

Figure 8. Post-intubation X-ray. Endotracheal tube in position. Widened mediastinum suspicious for mediastinal hematoma.

the mediastinum and create an artificial extra-pleural path for needle placement [1,4]. After creation of a safe extra-pleural window, a 22 gauge needle is removed and a large bore needle (18-gauge or larger) guide needle is advanced. By using a coaxial technique, fine-needle aspiration and core biopsies are performed through the guide needle $[1,4]$.

Infrequent complications of mediastinal biopsies include vasovagal reactions, air embolism, and tumor seeding [5]. Serious complications of CT guided mediastinal mass biopsy are unusual and deaths are very rare [6]. The most common life threatening complication of mediastinal biopsies include pneumothorax with incidence ranging from $27 \%$ $54 \%$ post-biopsy [5]. A large retrospective series placed incidence of pneumothorax at $20.5 \%$ and incidence of pneumothorax requiring chest drainage at $3.1 \%$ [7]. Other complications include pulmonary hemorrhage with or without hemoptysis, hemothorax, and chest wall hematoma [7]. In cases of hemothorax, chest tube drainage in acute phase is recommended followed by lytic therapy in subacute phase. Weinberg et al, reviewed a total of 830 thoracic biopsies from 20062011 and found a total of 4 out of $830(0.5 \%)$ developed post biopsy hemothoraces with ages ranging from 33-71. None of the patients had abnormal coagulation values or platelet counts and of note, one patient developed symptoms more than 12 hours later [8].Tomiyama, et al. in a survey of Japanese hospitals found only $0.092 \%$ of percutaneous lung biopsies resulted in hemothorax [9]. Yeowet, et al. reported only 1 out of $680(0.2 \%)$ coaxial biopsies to have complication of hemothorax[8].
Bleeding was reported to occur from pulmonary source or intercostal artery injury. No cases reported findings of injury to the pericardium resulting in cardiac tamponade.

In our case, the patient developed cardiac tamponade resulting from hemopericardium, a rarely reported complication of mediastinalbiopsies [10]. We postulate that the needle in our instance formed a small tear in the pulmonary vasculature and caused a communication between the pulmonary artery and the pericardium during tissue extraction. Fortunately, the diagnosis was made swiftly and the patient was taken for emergent exploratory median sternotomy.

\section{Conclusion}

Image guided percutaneous biopsy is routinely used for diagnosis of mediastinal lesions. Computed tomography (CT) and ultrasound guidance are used for precise localization of target lesion. CT imaging is established as the best imaging method for needle biopsy guidance [1]. Factors involved in deciding suitable approach include clinical circumstances, location, size of target lesion and comorbid conditions [2]. The best approach for percutaneous biopsy should be identified through review of prior images and history. It is imperative to recognize that risk of complications may vary depending on type of approach. Despite high diagnostic accuracy with rare incidence of complications, it is imperative to determine the safest access and be vigilant for any complication and provide prompt treatment when indicated.

\section{References}

1. Gupta S,Seaberg K, Wallace M (2005) Imaging-guided percutaneous biopsy of mediastinal lesions: different approaches and anatomic considerations. Radiographics 25: 763-786. [Crossref]

2. Kulkarni S, Kulkarni A, Roy D, Thakur MH (2008) Percutaneous computed tomography-guided core biopsy for the diagnosis of mediastinal masses. Annals of Thoracic Medicine 3: 13-17. [Crossref]

3. Westcott JL (1988) Percutaneous transthoracic needle biopsy. Radiology 169: 593-601

4. Langen HJ, Klose KC, Keulers P, Adam G, Jochims M, Gunther RW (1995) Artificial widening of the mediastinum to gain access for extrapleural biopsy: clinical results. Radiology 196: 703-706. [Crossref]

5. Hira L, Neyaz Z, Nath A, Borah S (2012) CT-guided percutaneous biopsy of intrathoracic lesions. Korean J Radiol 13: 210-226. [Crossref]

6. Bressler EL, Kirkham JA (1994) Mediastinal masses: Alternative approaches to CTguided needle biopsy. Radiology 191: 391-396. [Crossref]

7. Richardson CM, Pointon KS, Manhire AR, Macfarlane JT (2002) Percutaneous lung biopsies: a survey of UK practice based on 5444 biopsies. Br J Radiol 75: 731-735. [Crossref]

8. Weinberg, B, Watumull, L, Landay M, Omar, H (2013) Variable presentations of thoracic biopsy related hemothorac. Universal Journal of Clinical Medicine 1: 22-27.

9. Tomiyama N, Yasuhara Y, Nakajima Y, Adachi S, Arai Y, Kusumoto M, et al. (2006) CT-guided needle biopsy of lung lesions: a survey of severe complication based on 9783 biopsies in Japan. European Journal of Radiology 59: 60-64. [Crossref]

10. Protopapas Z, Westcott JL (2000) Transthoracic hilar and mediastinal biopsy. Radiol Clin North Am 38: 281-289.

Copyright: (C2017 Patel K. This is an open-access article distributed under the terms of the Creative Commons Attribution License, which permits unrestricted use, distribution, and reproduction in any medium, provided the original author and source are credited. 